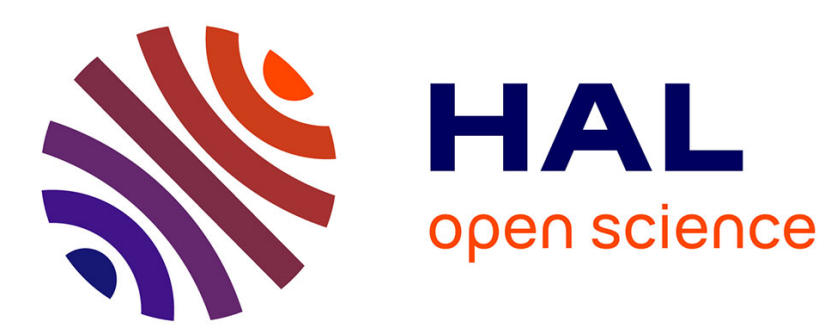

\title{
La fabrique parlementaire du discours bioéthique: analyse d'une arène de discussions -
}

\author{
M. Doumergue, N. Kalampalikis
}

\section{To cite this version:}

M. Doumergue, N. Kalampalikis. La fabrique parlementaire du discours bioéthique: analyse d'une arène de discussions -. Pratiques Psychologiques, 2020, 10.1016/j.prps.2020.03.003 . hal-02878327

\section{HAL Id: hal-02878327 \\ https://hal.univ-lyon2.fr/hal-02878327}

Submitted on 29 Jun 2020

HAL is a multi-disciplinary open access archive for the deposit and dissemination of scientific research documents, whether they are published or not. The documents may come from teaching and research institutions in France or abroad, or from public or private research centers.
L'archive ouverte pluridisciplinaire HAL, est destinée au dépôt et à la diffusion de documents scientifiques de niveau recherche, publiés ou non, émanant des établissements d'enseignement et de recherche français ou étrangers, des laboratoires publics ou privés. 


\title{
Dossier
}

\section{La fabrique parlementaire du discours bioéthique : analyse d'une arène de discussions}

\section{The parliamentary debates on bioethics in France: Analyzing an arena of discussions}

\author{
M. Doumergue*, N. Kalampalikis \\ Groupe de recherche en psychologie sociale (EA 4163), université Lumière Lyon 2, Bron, France
}

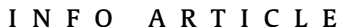

\section{Historique de l'article :}

Reçu le 4 juillet 2019

Accepté le 18 mars 2020

Disponible sur Internet le $\mathrm{xxx}$

\section{Mots clés :}

Bioéthique

Parlement

Anonymat

Don de gamètes

\section{R É S U M É}

Chaque révision de la loi relative à la bioéthique suscite un débat politique et social dans l'espace et la communication publics. Cet article se penche sur l'analyse de cette arène de discussions lors de la révision de la loi relative à la bioéthique de 2011, afin d'appréhender ce que les parlementaires ont mobilisé en termes de représentations sociales pour se positionner quant à ces questions socialement importantes. Au-delà de l'appréhension de la bioéthique, il se concentre sur la manière dont a été pensée et discutée la question de l'anonymat du don de gamètes dont le principe a été légalement maintenu en 2011, alors qu'il avait été au cœur de vives contestations publiques depuis le milieu des années 2000.

(C) 2020 Société Française de Psychologie. Publié par Elsevier Masson SAS. Tous droits réservés.

\section{A B S T R A C T}

Each revision of the law on bioethics triggers a political and social debate in public space and communication. This article examines the analysis of this arena of discourse during the second revision of the bioethics law in 2011, in order to observe what representational systems are involved by the members of the French parliament to think about and discuss on these socially important issues. More

\section{Keywords:}

Bioethics

Parliament

Anonymity

Gamete donation

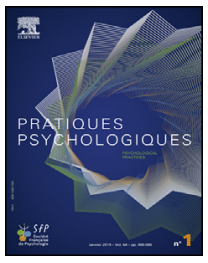


specifically, we focus on what may have led parliamentarians to decide in 2011 to maintain the anonymity of gamete donation, whereas this issue had been at the heart of intense public controversy since the mid-2000s.

(c) 2020 Société Française de Psychologie. Published by Elsevier Masson SAS. All rights reserved.

\section{Introduction}

En France, pour certains domaines, la consultation citoyenne est légalement prescrite. C'est notamment le cas pour la loi relative à la bioéthique pour laquelle la loi du 7 juillet 2011 prévoyait une révision par le Parlement dans un délai maximal de sept ans (désormais fixé à cinq ans), précédé de l'organisation d'États généraux confiée au Comité consultatif national d'éthique pour les sciences de la vie et de la santé (CCNE). Ainsi, les États généraux de la bioéthique se sont tenus pour la seconde fois au printemps $2018^{1}$. Avec le réexamen périodique de la loi, cela traduit le fait que la réflexion bioéthique est perçue comme devant s'actualiser régulièrement au fil des évolutions scientifiques, technologiques et sociétales (Bateman, 1998 ; Fox \& Swazey, 2008).

Cependant, ce n'est pas la sensibilité sociétale des questions posées par cette loi relative à la bioéthique qui a été avancée par le gouvernement pour expliquer les reports successifs de la troisième révision de la loi, initialement annoncée en 2018, finalement réalisée en octobre 2019. Celui-ci a mentionné plutôt l'encombrement législatif du début de la mandature. Cette latence a longtemps contrasté avec les signaux d'ouverture adressés, entre autres, par le rapport de la mission parlementaire sur la bioéthique, suite à l'avis du CCNE (2018). La mission parlementaire et le CCNE se sont tous deux déclarés favorables à l'utilisation post-mortem des gamètes, à l'autoconservation ovocytaire, en dehors de raisons médicalement constatées, à l'identification possible des dons de gamètes effectués suite à l'entrée en vigueur de la loi, etc. De l'ensemble des pratiques associées à la procréation médicalement assistée (PMA), seule la gestation pour autrui (GPA) demeurait l'objet d'une recommandation pour le maintien de son interdiction (CCNE, 2018).

Cette actualité de la révision de la loi française relative à la bioéthique, ainsi que l'intérêt et les débats qu'elle a suscités dans l'espace et la communication publics, nous a convaincus de nous tourner rétrospectivement vers les discussions parlementaires ayant eu lieu en 2011. Dans le cadre de ce numéro spécial consacré à l'analyse du discours, il apparaît en effet pertinent de se saisir de cette ade discussions afin d'appréhender comment les parlementaires débattent des questions socialement importantes qu'ils/elles ont à considérer dans l'exercice de la formation des lois. De quelle manière les parlementaires français ont pensé la bioéthique à l'occasion de la deuxième révision de la loi ? Quels éléments représentationnels ont été mobilisés pour ce faire ? Plus précisément, nous nous concentrerons sur l'anonymat du don de gamètes que les parlementaires ont majoritairement décidé de maintenir en 2011, alors qu'il avait fait l'objet de contestations ayant gagné en intensité dans l'espace public, depuis le milieu des années 2000.

\subsection{Contexte de l'étude : l'anonymat du don de gamètes au cœur de contestations publiques}

Si l'on considère le paysage légal international en matière d'encadrement des dons de gamètes, la France y fait figure d'exception à plus d'un titre. D'un point de vue institutionnel, les centres nationaux agréés, les Centres d'étude et de conservation des œufs et du sperme humains (CECOS), sont réunis depuis 1973 en une fédération qui assure l'organisation des dons et de leurs recours. Les premiers médecins et biologistes des CECOS se sont regroupés en tentant de répondre aux impératifs thérapeutiques et moraux du contexte social de l'époque. Ils ont ainsi organisé un modèle dit du don " de couple à couple " autour d'un système de principes " anonymat-gratuité-consentement », au sein duquel le

\footnotetext{
${ }^{1}$ Pas moins de 280 ateliers et débats ont été organisés et près de 30000 personnes ont déposé quelque 65000 contributions sur le site participatif dédié : https://etatsgenerauxdelabioethique.fr/.
} 
don est accessible aux couples de sexe différent souffrant d'une infertilité médicalement constatée (Bateman, 1994). Ces principes sont ceux qui ont été entérinés par les premières lois bioéthiques françaises de 1994. Du point de vue du contenu de la loi, l'exception résidait en ce que, jusqu'en 2015, les donneurs et donneuses de gamètes devaient déjà avoir procréé.

En ce qui concerne l'anonymat du don de gamètes, le paysage légal international a connu, ces 20 dernières années, une transformation marquée vers l'identification possible des donneurs et donneuses, à l'attention des personnes issues du don pouvant en faire la demande à leur majorité, dans la plupart des cas ${ }^{2}$. C'est dans ce contexte que la contestation publique quant à la stricte confidentialité des acteurs du don en France a pris place. La perspective de discuter et remanier en 2011 la loi relative à la bioéthique a mobilisé un ensemble d'acteurs des sphères scientifique, politique, religieuse et médicale et de la société civile. Les travaux et interventions publics d'un mouvement dit critique, réunissant des professionnels des sciences sociales (" psys », socio-anthropologues, juristes, etc.), proches d'associations de personnes issues de dons, se sont développés en préconisant l'ouverture de l'anonymat des dons de gamètes, au titre notamment du respect d'un droit d'accès des individus à leurs « origines personnelles » (Sebag-Depadt \& Delaisi de Parseval, 2010 ; Théry, 2010).

\subsection{Application de l'approche des représentations sociales aux discussions parlementaires}

L'approche théorique des représentations sociales (Moscovici, 2013; Jodelet, 2015) offre un cadre d'analyse pertinent pour l'étude de la pensée sociale autour d'un phénomène sensible et investi dans la sphère publique. L'anonymat du don de gamètes, mais aussi les définitions de la parenté, de la filiation, des origines, ont ainsi fait l'objet de préoccupations plurielles, ont suscité l'expression de points de vue personnels, juridiques, médicaux, socio-anthropologiques, psycho-psychanalytiques... Aussi, c'est en termes de rencontres de savoirs (Jodelet, 2013) que nous appréhendons ce qui est mobilisé dans la pensée sociale pour penser et se positionner quant à l'anonymat du donneur de gamètes. Cette rencontre de savoirs se traduit par des reconductions et des contestations quant aux significations à lui attribuer. Nous nous saisissons donc des discussions publiques à l'Assemblée nationale et au Sénat comme une arène de discussions où s'échangent et se confrontent différents savoirs (Van Dijk, 2003), c'est-à-dire comme un lieu de production et d'actualisation des représentations sociales du don de gamètes. Sa particularité tient notamment au fait que les discussions prennent place dans un contexte institutionnel et communicationnel contraint, dont l'issue (le vote) définit le légal et l'illégal. Nous tenons ces débats parlementaires comme reflet d'un état de société vis-à-vis d'un ensemble de questions bioéthiques.

Les représentations sociales ont une double fonction : permettre aux groupes sociaux de maîtriser leur environnement social, idéel et matériel; leur permettre de communiquer (Moscovici, 1976/2004). Il a été proposé que cette définition renvoie aux différents côtés du triangle (ego-alter-objet) du regard psychosocial (Moscovici, 1984), la fonction instrumentale impliquant principalement la relation ego-objet, alors que la fonction communicative concerne principalement la relation ego-alter (Gillespie, 2008).

Du point de vue de leur fonction instrumentale, les représentations sociales permettent à un groupe de répondre à un appel social à se positionner face à un objet non familier, c'est-à-dire présenté comme attentant au désirable ou à ce qui va de soi. Selon Wagner, Kronberger, et Seifert (2002), cet appel social correspond à une première phase de la dynamique de production et d'actualisation des représentations sociales, dite " de mise en controverse ". Deux phases lui succèdent (dites de " divergence " puis de " convergence ") lors desquelles le groupe accommode diversement l'objet non familier dans les répertoires existants de connaissances sociales, pour le rendre intelligible, puis fait converger l'environnement symbolique ambigu ainsi créé, par l'élimination de certaines interprétations. Ce faisant, le groupe se dote d'une nouvelle interprétation conventionnalisée qui lui permet de se positionner d'une manière cohésive, renforçant ainsi son identité et son histoire. Ainsi, le groupe

\footnotetext{
${ }^{2}$ Cette transformation a fait entrer une seconde série de pays (ex. Royaume-Uni, 2005 ; Finlande et Belgique, 2007) dans le rang de ceux ayant opté pour un don de gamètes identifiable, rejoignant ainsi les membres d'une première série, constituée quant à elle dès les années 1980 (ex. Suède, 1985 ; Allemagne, 1989 ; Autriche, 1992).
} 
actualise et agence des significations préexistantes d'une manière qui lui est propre et qui, une fois (trans)formées en représentation sociale, est ce par quoi les individus se reconnaissent membres de celui-ci ${ }^{3}$.

Cette formalisation de la dynamique représentationnelle (production et actualisation de représentations) est congruente avec celle présentée par Castro (2012) pour rendre compte des effets des modifications de lois, en termes de résistances et de changements publics. À l'échelle de la réception d'une loi, Castro (2012) propose que, pour qu'une nouvelle loi remplisse son rôle, les représentations qu'elle promeut doivent devenir hégémoniques. Selon la définition des représentations hégémoniques (Moscovici, 2013), les valeurs et les buts sociaux génériques exprimés par le cadre légal doivent devenir structurants pour le groupe nation, d'une manière uniforme et coercitive. Selon Castro (2012), cela passe par une phase de transition lors de laquelle les représentations relatives à la loi peuvent être davantage décrites comme émancipées : les différents groupes impliqués proposent des versions divergentes des valeurs génériques et poursuivent des buts divergeant les uns des autres (Moscovici, 2013). Pour l'auteure, lors de cette phase, la traduction de la loi en pratiques concrètes dans des contextes spécifiques est intensément négociée dans les communications publiques et les interactions quotidiennes. Néanmoins, il a été montré (Mouro \& Castro, 2012) que les acteurs sociaux étaient soucieux de ne pas ouvrir de conflit sous formes de confrontation et de polarisation, propres aux représentations polémiques, afin de maintenir de la coopération entre les différents groupes et de garder ouverte la négociation des significations. Pour ce faire, les idées divergentes, contestant la loi dans son application locale, étaient exprimées de manière simultanée avec un accord conventionnalisé quant aux valeurs générales de la loi (réaffirmées mais non discutées), maintenant les représentations sociales impliquées dans un état polyphasique (Mouro \& Castro, 2012).

Du point de vue de leur fonction communicative, les représentations sociales fournissent une base commune et conventionnelle suffisante (c.-à-d. des systèmes de savoirs suffisamment partagés) pour rendre la communication possible. Dans un article soulignant l'importance de l'étude de la dimension pragmatique de la communication pour l'étude des représentations sociales, Moscovici (1994, p. 168) pointe que, sous cette forme conventionnalisée, il est loisible de tenir " les représentations sociales comme des présuppositions enfouies sous les couches de mots et d'images qui flottent dans l'esprit des gens ». En d'autres termes, allant de soi, ces représentations ne sont que partiellement explicitées dans la communication sémantique, et font surtout l'objet d'inférences pragmatiques par les interactants. C'est que, dans bien des cas, l'enjeu pour ceux-ci est de conserver un (faux) consensus qui permette la poursuite des interactions (le maintien du lien ego-alter). Il n'en reste pas moins que les représentations ne peuvent être tenues pour " complètement distribuées ", mais sont bien plutôt " partiellement partagées » entre les groupes et les interactants (Moscovici, 1994, p. 168). Ce sont les écarts entre les connaissances, et les divergences entre les savoirs, qui rendent d'ailleurs la communication nécessaire. Le contenu des représentations sociales s'explicite alors dans les discours. Les limites du partage des représentations supposées communes se font jour, il y a " mise en controverse » (Wagner et al., 2002), au risque des divergences et des dissensions.

Autrement dit, les représentations sociales sont produites et communiquées à deux niveaux, au niveau de la communication pragmatique et au niveau de la communication sémantique : elles peuvent être implicites dans le contexte de communication et elles peuvent être explicites dans le contenu de la communication. "Elles sont communiquées dans le contexte quand elles sont utilisées comme des moyens de communiquer et elles sont [communiquées] dans le texte [ce qui est dit] lorsque les représentations en elles-mêmes deviennent l'objet de la conversation » (Gillespie, 2008, p. 378).

Dans un article de 2005, Kalampalikis et Moscovici proposent de s'intéresser aux traces indirectes de ces communications dans le vocabulaire. Pour ce faire, ils invitent à prendre en compte dans l'analyse lexicométrique, non seulement une interprétation sémantique mais aussi pragmatique d'indices relevant du lexique, en renseignant la température informationnelle de corpus discursifs (dans leur cas). Brièvement, nous pouvons dire que l'hypothèse de la température informationnelle pose qu'à volume égal, un texte présentant une grande redondance lexicale est peu informatif - mais vise davantage à

\footnotetext{
${ }^{3}$ L'approche genevoise des représentations sociales opérationnalise par un outil statistique (l'analyse factorielle) cet ancrage des représentations sociales dans les groupes sociaux (cf. Clémence, Doise, \& Lorenzi-Cioldi, 1994).
} 
Tableau 1

Corpus : première lecture du projet de loi relatif à la bioéthique (loi nº 2011-814 du 7 juillet 2011).

\begin{tabular}{lll}
\hline Chambre & Type d'échanges & Séance \\
\hline Assemblée & Discussion générale & $1^{\text {re }}$ et $2^{\mathrm{e}}$ séances du 08.02.2011 et du 09.02.2011 (13 h) \\
nationale & Articles 14 à 20 & $2^{\mathrm{e}}$ et $3^{\mathrm{e}}$ séances du $10.02 .2011(3 \mathrm{~h})$ \\
Sénat & Discussion générale & Séance du 05.04.2011 $(4 \mathrm{~h})$ \\
& Articles 14 à 20 & Séance du 07.04.2011 $(1 \mathrm{~h})$ \\
\hline
\end{tabular}

communiquer - tandis qu'un texte contenant une grande variété lexicale sera hautement informatif et davantage à même de faire valoir des savoirs (Kalampalikis \& Moscovici, 2005). Précédemment, Moscovici (1967), dans une recherche sur les processus de communication et de langage, a éprouvé expérimentalement la température informationnelle comme une bonne mesure du degré de pression à la référence ou à l'inférence. Rappelons qu'une pression à l'uniformité peut se faire parfois par une pression à la référence (quant à ce dont on parle), parfois par une pression à l'inférence sur les opinions et jugements des membres d'un groupe. Dans le premier cas, la fonction instrumentale des représentations sociales est davantage utilisée et le lien ego-objet est principalement impliqué. Nous nous attendons à ce que la température informationnelle soit plus élevée que dans le second cas, où le lien ego-alter est principalement concerné et où la fonction communicative des représentations sociales préférentiellement utilisée (Kalampalikis \& Moscovici, 2005).

Dans cet article, nous étudions une arène de discussions particulière, dans laquelle la " mise en controverse " d'objets émergents (Wagner et al., 2002) est institutionnellement et régulièrement prescrite au groupe concerné : les discussions parlementaires de la révision de la loi française relative à la bioéthique en 2011. L'objectif principal de l'étude, fondée sur une analyse lexicométrique (Ratinaud \& Marchand, 2015), consiste en la mise en évidence des représentations sociales impliquées par les parlementaires pour penser la bioéthique et la question socialement sensible de l'anonymat du don de gamètes. Nous nous intéressons aux types de savoirs et aux catégories de pensée auxquelles ces discussions réfèrent pour leur permettre de se positionner quant à ces enjeux.

Du point de vue d'objectifs plus spécifiques, nous examinerons si l'organisation des discussions est marquée par un processus de conventionnalisation. Celui-ci rendrait compte d'une visée des discussions parlementaires qui consisterait en l'élaboration collective des représentations hégémoniques qui seront ensuite promues par le texte de loi. Il est à noter, dans le cas de la loi relative à la bioéthique de 2011, que cette pression à l'uniformité encadrant les discussions parlementaires peut avoir été soutenue par une absence de consignes de votes de la part des groupes parlementaires. Plus localement, nous observerons ce qui fait consensus et ce qui fait polémique dans les discussions, en nous attendant à ce que les représentations davantage émancipées ou polémiques s'ancrent dans les groupes politiques des parlementaires, et fassent l'objet de discours à la température informationnelle plus élevée que les représentations hégémoniques/conventionnalisées.

\section{Méthodologie : analyse lexicométrique des discussions parlementaires de la loi relative à la bioéthique (2011)}

Le corpus est constitué des retranscriptions des discussions en séances publiques à l'Assemblée nationale et au Sénat, établies par les rédacteurs des débats et publiées au Journal Officiel. Au sein de la première lecture du projet de loi, nous avons retenu les prises de parole issues des discussions générales et de la défense des amendements déposés pour les articles de lois relatifs au don de gamètes (articles 14 à 20 relevant des titres V et VI « Anonymat du don de gamètes » et « assistance médicale à la procréation », voir Tableau 1). Ce corpus combine les niveaux de généralité. Il permet ainsi de présenter la discussion centrée sur le don de gamètes, insérée dans le contexte général des débats parlementaires quant à la bioéthique (discussions générales), tout en accédant également à sa spécificité (articles 14 à 20). 
Ce volumineux corpus a fait l'objet d'une analyse lexicométrique grâce au logiciel Iramuteq (Ratinaud, 2014) mettant en œuvre la méthode Alceste (Reinert, 1999)4. L'objectif de cette méthode, basée sur une classification descendante hiérarchique (CDH), est l'organisation topique du discours à travers la mise en évidence de "mondes lexicaux " (Kalampalikis, 2003). La mise en évidence des traces lexicales les plus importantes du corpus permet de révéler les mondes lexicaux organisant ce corpus. Le logiciel ne s'intéresse pas au sens des mots, mais à l'identification de " patterns répétitifs de langage " qui font " lieux communs ». L'hypothèse sous-jacente est qu'il est possible de considérer l'utilisation d'un vocabulaire spécifique comme un moyen de détecter différentes manières de penser un objet, puisque différents points de références produisent ces différentes manières de penser (Kronberger \& Wagner, 2003). Il s'agit donc de cartographier les principaux « lieux communs » d'un discours.

Le travail d'interprétation est guidé ensuite par ce " commun " constituant chaque lieu/classe. L'interprétation de chaque classe est aidée par les mots significativement présents et absents dans les segments de texte caractéristiques de chacune (la force de la typicalité est calculée par un $\chi^{2}$ ). Le travail d'interprétation est également guidé par les différences entre chaque classe, appréhendables via le dendrogramme (ou la projection du contenu sur un plan factoriel).

De plus, il est possible de tester un certain nombre d'hypothèses en définissant préalablement pour chaque texte ou pour chaque tour de parole, des variables externes (la force de la typicalité est à nouveau calculée par un $\chi^{2}$ pour chaque classe).

En ce qui concerne le présent corpus, quatre variables ont été considérées :

- le/la parlementaire : 113 modalités : chaque tour de parole a été encodé avec un numéro attribué à chaque parlementaire ;

- la séance parlementaire : 12 modalités : numéro et date de la séance (ex. $1^{\text {re }}$ séance 08/02);

- le type d'échanges : 8 modalités : discussion générale vs des articles de loi (articles 14 à 20);

- la Chambre parlementaire : 2 modalités : Assemblée nationale vs Sénat.

La première variable permet de considérer l'hypothèse d'un ancrage des représentations dans les groupes politiques. Le cas échéant, il est attendu que plusieurs parlementaires relevant d'une même appartenance politique soient significativement associés à une même classe, ou à un même ensemble de classe, distincts d'autres classes ou ensemble de classes associés à plusieurs parlementaires relevant d'une autre appartenance politique.

La deuxième variable permet de considérer l'hypothèse diachronique d'un changement de représentations à mesure de l'avancée des débats (cf. processus de conventionnalisation).

Les deux autres variables permettent de contrôler l'influence de l'organisation institutionnelle des discussions parlementaires sur la structuration des discours.

Enfin, afin de renseigner " la température informationnelle » des classes (Kalampalikis \& Moscovici, 2005), nous nous sommes intéressés à certains indices relevant du lexique (ex. hapax, indice de diversité, indice de rareté ; cf. Appendice, Tableau 2). Plus particulièrement, nous considérons, de manière relative entre les classes, que des indices de diversité (type/token ratio - TTR) et de rareté (IR) peu élevés traduisent une faible variété et une faible richesse du vocabulaire d'une classe et donc une basse température informationnelle de celle-ci (le vocabulaire y étant fortement redondant). De sorte que des indices de diversité et de rareté élevés sont la marque d'une forte température informationnelle pour une classe, caractérisée ainsi par un vocabulaire davantage varié et riche (Kalampalikis \& Moscovici, 2005).

\footnotetext{
${ }^{4}$ La méthode repose sur un découpage du corpus en fragments de taille analogues, dits " unités de contexte élémentaires " (uce ou segments de texte). Ce découpage se fait au sein "d'unités de contexte initiales " (uci ou textes) définies par l'utilisateur. Ensuite, le logiciel lemmatise les mots en formes (suppression des pluriels, conjugaisons...). Un tableau de contingence indique la présence ou l'absence de ces formes au sein de chaque unité ou segment de texte. Une classification descendante hiérarchique $(\mathrm{CDH})$ est appliquée sur celui-ci, séparant les segments de texte en fonction de leurs contrastes de lexique. Les segments de texte sont répartis d'abord en deux classes en fonction de la distribution du vocabulaire, puis ces deux classes sont divisées à nouveau jusqu'à ce qu'un nombre de classes stable soit atteint. Le résultat est une organisation des classes (se présentant sous forme de dendrogramme), à savoir les « mondes lexicaux » ou « lieux communs de discours » présents dans le corpus.
} 


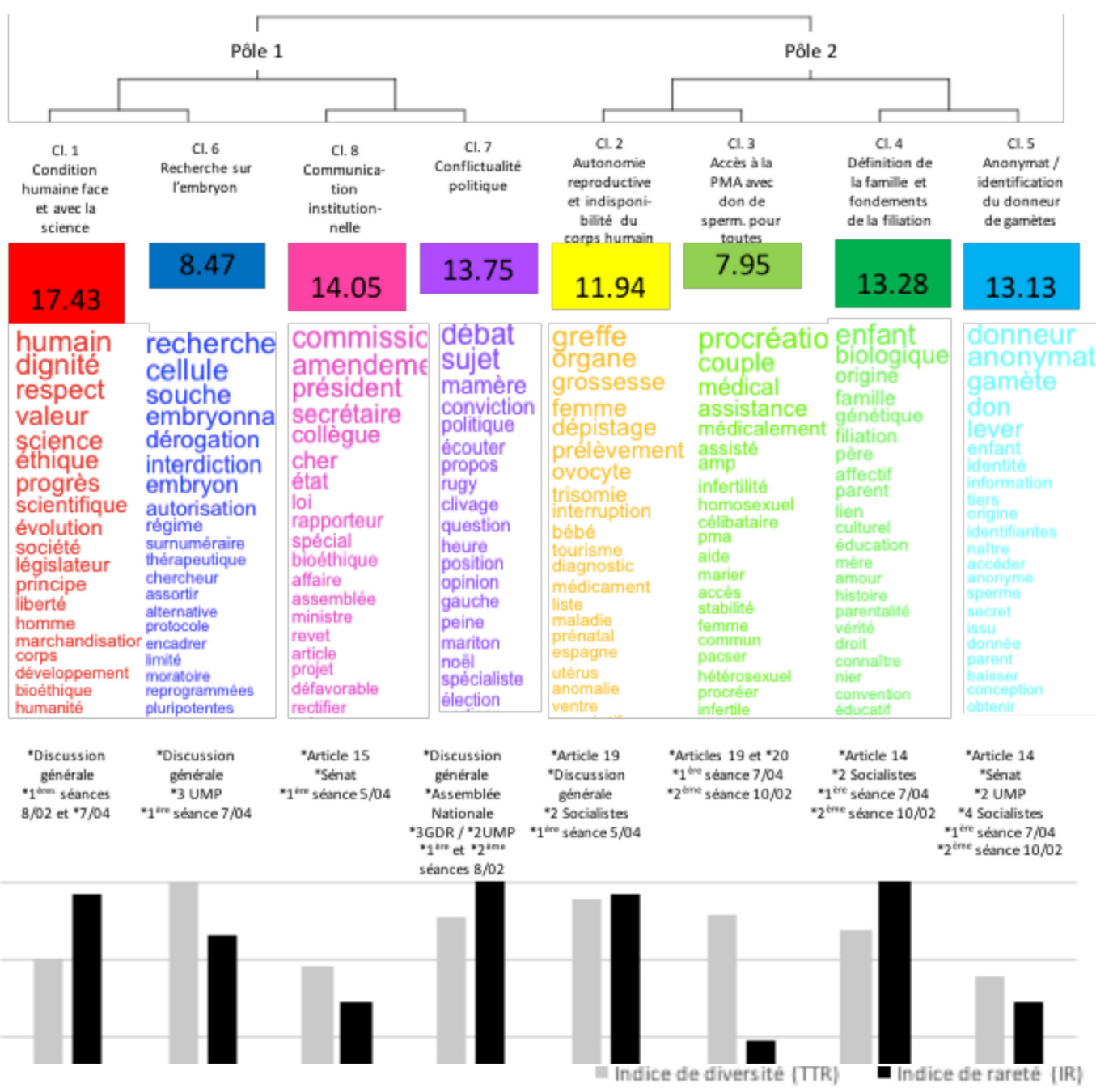

Fig. 1. Classification descendante hiérarchique $(\mathrm{CDH})$ du corpus des discussions parlementaires avec indicesde la température informationnelle des classes.

Croisés avec l'analyse du contenu des mondes lexicaux et les variables externes associées, ces indices informent l'usage des représentations conventionnalisées/hégémoniques (fonction communicative des représentations sociales) ou davantage polémiques (fonction instrumentale des représentations sociales) dans l'exercice de conviction et de justification que sont les débats parlementaires.

\section{Résultats}

La classification descendante hiérarchique (CDH, 94,5\% du corpus analysé) met en évidence deux pôles comportant chacun quatre classes lexicales. La Fig. 1 présente les titres attribués aux classes, ainsi que la part du corpus qu'elles représentent. Elle indique aussi les mots les plus fréquemment associés à chaque classe ainsi que les variables externes significativement représentatives de chacune. Enfin, certains indices relevant du lexique, calculés afin de rendre compte de la température informationnelle 
de chaque classe, sont présentés de manière tronquée et normalisée afin de faire ressortir la variabilité entre les classes.

La première partition du corpus l'a scindé en deux : le premier pôle $(53,8 \%$ - classes $1,6,7$ et 8$)$ rassemble surtout des classes caractéristiques des discussions générales du projet de loi. Le second pôle (46,2 \% - classes $2,3,4$ et 5 ) regroupe des classes qui réfèrent aux défenses des amendements déposés, concernant les articles de loi encadrant des techniques particulières (articles 14,15, 19 et 20). Les autres variables externes apparaissent bien moins rendre compte de la structuration du discours. L'association des séances parlementaires pour chaque classe est redondante avec celle des modalités de la variable "type d'échanges " (discussion générale vs articles). Les discussions ayant eu lieu au Sénat sont caractéristiques d'une classe dans chaque pôle, celles à l'Assemblée nationale d'une classe dans le premier pôle. Cinq classes sur les 8 sont caractérisées par plus de deux parlementaires de la même appartenance politique et seules 2 classes (une dans chaque pôle - classes 5 et 7 ) sont significativement associées à plus de 2 parlementaires de deux groupes politiques différents. Le contenu des classes réparties en deux pôles, ainsi que l'interprétation des indices relevant du lexique calculés, sont détaillés ci-après.

\subsection{Pôle 1 : légiférer en matière bioéthique}

Au sein du premier pôle : la classe 1 est la plus volumineuse du corpus $(17,43 \%)$ et est celle où les parlementaires pensent le rôle du législateur en matière bioéthique $\left(\chi^{2}=121,83, p<.001 ; \chi^{2}=53,75\right.$, $p<.001)^{5}$. Ce rôle consiste à préserver la condition humaine face et avec la science $\left(\chi^{2}=208,84\right)$ :

"C'est l'humanité même de l'Homme que les progrès de la science et de la technique questionnent. Il est urgent de déterminer si ces bouleversements scientifiques accroîtront la servitude des hommes ou au contraire garantiront leur liberté, c'est-à-dire leur puissance d'agir " (Assemblée nationale, $1^{\text {re }}$ séance $09 / 02$, discussion générale).

Il s'agit d'encadrer légalement les évolutions scientifiques, à partir de principes axiologiques et moraux fondamentaux : le respect de la dignité humaine et le refus d'une marchandisation du corps humain $\left(\chi^{2}=139,85 ; 228,54 ; 257,07 ; 267,94 ; 73,41\right)$.

"Ces principes communs à un grand nombre de sociétés sont assez simples : respect de la vie et de la dignité humaine, non-instrumentalisation du corps humain, et en un mot vigilance face à des apprentis sorciers prêts à tout au nom de la science " (Assemblée nationale, $2^{\mathrm{e}}$ séance 08/02, discussion générale).

Du point de vue des indices relevant du lexique, nous constatons une température informationnelle relativement basse. En effet, malgré un vocabulaire hétérogène (indice de rareté élevé ; $\mathrm{IR}_{\mathrm{cl} 1}=.028 \epsilon$ $[.017, .029])$, cette classe est marquée par une forte redondance du lexique (faible indice de diversité ; $\left.\mathrm{TTR}_{\mathrm{cl} 1}=.135 \in[.128, .169]\right)$. Enfin, du point de vue d'autres indices, il est à noter la présence significative des pronoms personnels et possessifs à la première personne du pluriel ( " nous "; " notre "; "nos » : $\left.\chi^{2}=39,90 ; 31,22 ; 29,69\right)$. L'analyse de ces indices soutient notre interprétation de cette classe comme un lieu lexical où les parlementaires réaffirment des principes communs pour penser la bioéthique et le rôle du législateur français à cet égard : " Deux principes intangibles guident nos travaux, le respect de la dignité de la personne humaine et l'interdiction de la marchandisation. C'est bien évidemment la colonne vertébrale, le fil rouge et ce qui doit être l'esprit de la loi " (Sénat, $1^{\text {re }}$ séance $05 / 04$, discussion générale).

La classe 6 est l'une des plus petites du corpus (8,5\%). Elle réfère à la recherche sur l'embryon et les cellules embryonnaires $\left(\chi^{2}=1346,88 ; 853,81 ; 1335,69 ; 1033,24\right)$, dont la fin du moratoire a précipité la révision de la loi relative à la bioéthique. Cette classe comprend de manière caractéristique un champ lexical relatif aux biotechnologies (cellules pluripotentes; $\chi^{2}=86,63$ ) et au protocole scientifique $\left(\chi^{2}=96,68\right)$. Cela se traduit par une température informationnelle très élevée (indice de diversité le plus haut des classes : $\operatorname{TTR}_{\mathrm{cl6}}=.169 \in[.128, .169]$; indice de rareté élevé : $\left.\operatorname{IR}_{\mathrm{cl6}}=.025 \in[.017, .029]\right)$.

${ }^{5}$ Les $\chi^{2}$ indiqués dans cet article sont tous significatifs à $p<.001$. 
Il s'y discute le bien-fondé d'une autorisation encadrée de la recherche versus celui d'une interdiction assortie de dérogations :

" Il n'y aurait pas de différence entre ce principe et celui de l'autorisation encadrée puisqu'il ne gênerait en rien les progrès de la recherche et respecterait les convictions attachées à la dignité de l'embryon humain » (Assemblée nationale, $1^{\text {re }}$ séance 09/02, discussion générale).

La distinction entre les classes 1 et 6 , d'une part, et les classes 7 et 8, d'autre part, renvoie à une distinction entre fond et forme. En effet, les classes 7 et 8 renvoient aux formes de la rhétorique parlementaire.

La classe 8 contient des mots qui servent de liant de la communication institutionnelle lors de l'activité de préparation des lois. Cette fonction est corroborée par une température informationnelle particulièrement basse (faible indice de diversité : $\operatorname{TTR}_{\mathrm{cl} 8}=.132 \in[.128, .169]$; faible indice de rareté : $\left.\mathrm{IR}_{\mathrm{cl} 8}=.20 \in[.017, .029]\right)$.

La classe 7 est caractéristique de la discussion générale de l'Assemblée nationale, et est caractérisée par les prises de parole de membres de deux groupes parlementaires ( 3 issus de la Gauche Démocrate et Républicaine - GDR -, et 2 de l'Union pour un Mouvement Populaire - UMP). Leur nom est présent en tant que formes constitutives de la classe (ex. Mamère, De Rugy, Mariton - $\chi^{2}=148,06$; 79,82 ; 68,38 ), et ces interpellations traduisent une situation particulière d'interlocution au sein de la discussion générale ${ }^{6}$. En termes de température informationnelle, cela se traduit par un indice moyen de diversité $\left(\operatorname{TTR}_{\mathrm{cl} 7}=.153 \in[.128, .169]\right)$ et un fort indice de rareté $\left(\operatorname{IR}_{\mathrm{cl} 7}=.029 \in[.017, .029]\right)$. La présence significative des pronoms personnels et possessifs à la deuxième personne du pluriel est à souligner ( "vous "; " vôtres "; " votre »; " vos » : $\chi^{2}=107,00 ; 18,53 ; 15,46 ; 15,32$ ).

L'examen des segments de texte caractéristiques de la classe indique qu'ils relèvent essentiellement de la défense de deux motions déposées par le groupe GDR, demandant respectivement le rejet préalable du projet de loi et son renvoi en commission, ainsi que des interruptions et des réponses dont cette défense a fait l'objet ${ }^{7}$.

Nous interprétons ainsi cette classe comme un lieu de conflictualité politique.

La notion de politique $\left(\chi^{2}=98,49\right)$ y revêt d'ailleurs plusieurs acceptions. Elles transparaissent alors que se discutent explicitement ce qui devrait fonder les décisions en matière bioéthique, et la nature que devrait avoir ce débat $\left(\chi^{2}=234,69\right)$.

La question de la représentation politique et du rôle du législateur est reposée explicitement, mais en relation avec les autres sphères de la vie publique : les spécialistes ou l'opinion publique ont-ils été suffisamment écoutés ? $\left(\chi^{2}=67,71 ; 72,74 ; 89,97\right)$.

Une utilisation politicienne-électoraliste du réexamen des lois bioéthiques de la part du gouvernement est dénoncée, rappelant le contexte d'élections présidentielles à venir. Le statu quo aurait été promu afin de satisfaire les plus conservateurs de la majorité $\left(\chi^{2}=61,33 ; 30,24\right)$.

L'idée selon laquelle les débats bioéthiques relèvent du politique-idéologique est parfois défendue.

"Ce débat dépasse les clivages politiques traditionnels ce qui n'exclue pas qu'il ne soit pas aussi politique (...) » ; " Faisant appel aux principes éthiques et aux convictions philosophiques ou religieuses autant qu'à l'engagement politique, la réflexion de chacun transcende les clivages partisans " (Assemblée nationale, $2^{\mathrm{e}}$ séance 09/02, discussion générale).

A contrario, il est argué que les questions bioéthiques relèvent de l'intime, et que les décisions à leur égard doivent être prises en conscience, selon les convictions de chacun $\left(\chi^{2}=26,73 ; 48,85 ; 116,54\right)$.

La réaffirmation du fait que les sujets bioéthiques dépassent les clivages politiques-partisans est particulièrement prégnante $\left(\chi^{2}=212,81 ; 79,82 ; 60,71\right)$.

\footnotetext{
${ }^{6}$ Au sein de discussions générales, les prises de parole sont quelque peu " déconnectées " les unes des autres dans l'interlocution, puisqu'elles sont souvent préparées, voire écrites, en amont des séances.

7 « C'est un sujet trop important et trop délicat pour que chacun n'agisse pas avant tout en vertu de sa conscience. Je regrette que le rapporteur, le ministre et certains représentants de groupes aient cédé à la tentation de la caricature dans leur réponse à la motion de Noël Mamère " (Assemblée nationale, $1^{\text {re }}$ séance 08/02, discussion générale). " Nous proposerons le rejet de cette motion, mais je pense que notre collègue De Rugy a très bien introduit le sujet. Au fond, comme il l'a dit très justement, notre débat relève de l'intime » (Assemblée nationale, $2^{\mathrm{e}}$ séance 08/02, discussion générale).
} 
"Ce débat est singulier parce qu'il nous incite à sonder notre conscience et à dépasser évidemment les réflexes partisans habituels. Quelles que soient notre sensibilité et notre appartenance politique nous savons bien qu'une appartenance commune plus profonde, plus large aussi, nous rassemble » (Sénat, $1^{\text {re }}$ séance 07/04, discussion générale).

Enfin, l'euthanasie et la peine de mort $\left(\chi^{2}=61,33 ; 30,24\right)$ sont parfois citées comme des précédents en termes d'affrontements politiques-idéologiques ou comme des exemples passés d'une réflexion élaborée communément par les parlementaires :

"Je crois pourtant utile de préciser que l'on ne peut pas attendre un hypothétique et illusoire consensus sur tous ces sujets de bioéthique. Rappelons que les débats sur l'avortement, la pilule, la peine de mort ou plus récemment le pacs n'ont jamais été consensuels "; " Il y eut bien sûr l'abolition de la peine de mort, la loi sur l'avortement et plus proche de nous cette loi de 2004 qui touche précisément notre sujet sans compter la loi sur la fin de vie " (Assemblée nationale, $2^{\mathrm{e}}$ séance $08 / 02$, discussion générale).

\subsection{Pôle 2 : don, famille, corps, sexualité}

Le second pôle rassemble, d'une part, les classes 2 et 3 caractéristiques des articles de loi relevant du titre VI " Assistance médicale à la procréation », et d'autre part, les classes 4 et 5 caractéristiques de l'article 14 relevant du titre V "Anonymat du don de gamètes ". Chaque binôme comprend une classe à la température informationnelle plutôt basse et une classe à la température informationnelle davantage élevée.

La classe 3 est surreprésentée dans les discussions des articles 19 et 20 qui définissent entre autres les conditions d'accès à la PMA avec don de sperme. Cette classe est un lieu très homogène du point de vue du lexique, en ce qu'elle comporte le plus faible indice de rareté parmi les lieux lexicaux $\left(\mathrm{IR}_{\mathrm{cl} 3}=.17 \in[.017, .029]\right)$. La discussion coulisse le long d'une opposition médical-sociétal. À l'un des pôles de l'opposition, l'assistance médicale à la procréation (AMP) est conçue comme solution à une infertilité médicalement constatée, à l'autre, l'ouverture de l'AMP pour toutes est prônée, c'est-à-dire également aux femmes célibataires et aux couples homosexuels ( $\chi^{2}=620,76 ; 1070,18 ; 315,31 ; 437,3$; 836,$10 ; 248,85$ ). La redondance lexicale particulièrement forte peut s'expliquer par le fait que cette dernière prise de position reprend cette opposition, en appelant à ouvrir l'accès à cette pratique sans discrimination ou distinction $\left(\chi^{2}=58,07 ; 19,69 ; 15,91\right)$ :

« Nous proposons par cet amendement d'ouvrir l'accès à l'assistance médicale à la procréation aux couples de femmes et aux femmes célibataires en âge de procréer que l'infertilité soit médicale ou qu'elle soit sociale " (Sénat, $1^{\text {re }}$ séance $07 / 04$, article 20).

La classe 2 , quant à elle, rassemble un ensemble composite de thèmes (don et greffe d'organes, interruption volontaire de grossesse, GPA, bébé-médicament $-\chi^{2}=46,81 ; 270,36 ; 232,11 ; 195,51 ; 38,78$; $111,88)$, provenant préférentiellement des discussions générales. Cet ensemble se traduit par une haute température informationnelle $\left(\mathrm{TTR}=.161 \in[.128, .169] ; \mathrm{IR}_{\mathrm{cl} 2}=.028 \in[.017, .029]\right)$. Ces thèmes viennent y faire classe commune avec celui traité par l'article 19 : l'éligibilité au don de gamètes. La question plus précise est celle d'ouvrir aux femmes n'ayant pas procréé la possibilité de donner leurs ovocytes $\left(\chi^{2}=191,06 ; 166,67\right)$. Il s'agit de réduire les listes d'attente en France et le tourisme procréatif des couples infertiles vers des pays où il serait fait commerce des gamètes $\left(\chi^{2}=81,29 ; 94,78\right)$. Une autoconservation des gamètes est de plus prévue pour les donneuses en vue de pallier un faible risque d'infertilité induit par la ponction du don. L'utilisation personnelle ultérieure de cette autoconservation est discutée par les parlementaires en opposant les notions d'autonomie reproductive et d'indisponibilité du corps humain : à quelles conditions serait-on encore dans le cadre de la générosité d'un don volontaire ou déjà dans celui d'une marchandisation d'éléments du corps humain ?

«Certes [la motivation] n'est pas monétaire mais elle est peut-être encore plus intéressante. Bien sûr on encadrerait l'utilisation de cet ovocyte conservé en la limitant aux cas d'infertilité qui auraient pu être provoqués par un prélèvement "; "Dans un principe de générosité et d'autonomie, je donne pour moi, mais en réalité, je ne donne pour moi que si je suis malade, je 
ne suis alors pas dans une éthique d'autonomie » (Assemblée nationale, $2^{\mathrm{e}}$ séance 10/02, article 19).

Les discussions de l'article 14, relatif à l'anonymat des dons de gamètes, sont caractéristiques des classes 4 et 5 . La classe 5 a une température informationnelle basse et est marquée par un lexique peu étendu (TTR $=.128 \in[.128, .169])$ et particulièrement stéréotypé $\left(\operatorname{IR}_{\mathrm{cl} 5}=.020 \in[.017, .029]\right)$. Elle est dévolue à la présentation des amendements aux articles de loi en faveur de l'anonymat ou de l'identification du donneur et aux dispositifs afférents. Une levée de l'anonymat du donneur de gamètes permettrait aux enfants nés de son don d'accéder à des informations identifiantes le concernant $\left(\chi^{2}=641,13 ; 839,61 ; 847,61 ; 701,87 ; 277,3 ; 161,63 ; 144,57\right)$. Une autre possibilité débattue dans les chambres est d'envisager qu'à sa majorité, l'adulte souhaitant accéder à ses origines puisse solliciter le géniteur $\left(\chi^{2}=123,05 ; 151,65 ; 46,69\right)$, via une institution spéciale, pour recueillir son consentement à se faire connaître. Notons l'association significative à la classe 5 de membres de groupes provenant des deux tendances majeures du paysage politique français de l'époque (UMP et PS).

Alors que le vocabulaire de la classe 5 s'attache préférentiellement au donneur, et relève du concret et du situé, la classe 4 est le lieu lexical de l'enfant, du père et de la mère $\left(\chi^{2}=458,67 ; 163,25 ; 86,2\right)$, où l'abstrait et le conceptuel prévalent. Ce qui domine au sein de cette classe, ce sont les notions de la famille, de filiation, de parentalité $\left(\chi^{2}=222,18 ; 190,12 ; 73,09\right)$. La nature de ces liens est à qualifier : biologique, génétique, affectif, culturel, éducatif, etc. $\left(\chi^{2}=134,64 ; 340,8 ; 191,04 ; 160,47 ; 103,3 ; 57,56\right)$.

" Lever l'anonymat risquerait de briser ou de fragiliser cette filiation sociale au bénéfice de la filiation génétique avec des conséquences que les familles comme les donneurs redoutent tant " (Assemblée nationale, $2^{\mathrm{e}}$ séance $08 / 02$, discussion générale)

"Au nom de quoi faudrait-il subitement effacer ces données cela me semble contraire à l'intérêt de l'enfant dont la filiation est autant biologique que sociale environnementale ou affective " (Sénat, $1^{\text {re }}$ séance $07 / 04$, article 14 )

"Gardons toujours à l'esprit une vision équilibrée de la famille à la fois réalité biologique si importante aux yeux de l'enfant et lieu d'éducation pour sa construction sociale point de vue privilégié par les parents » (Sénat, $1^{\text {re }}$ séance 05/04, discussion générale).

Des professionnels sont cités à titre d'experts des champs relatés - psychologues et psychanalystes, ainsi que sociologues $\left(\chi^{2}=19,34 ; 32,67 ; 18,98\right)$. Une autre référence mobilisée est celle des conventions internationales et du Droit $\left(\chi^{2}=58,14 ; 51,60 ; 71,71\right)$.

« On ne peut prétendre que permettre à l'enfant de connaître ses origines génétiques, c'est faire le choix d'une parenté biologique. Puisque même le droit nous dit le contraire, cela ne changera rien " (Assemblée nationale, $1^{\text {re }}$ séance 09/02, discussion générale)

"C'est du reste ce que les conventions internationales ont affirmé et réaffirmé en donnant à l'enfant le droit de connaître ses origines et pourquoi l'ont-elles fait ? Parce que l'on considère qu'une personne est un tout affectif, social, biologique " (Assemblée nationale, $2^{\mathrm{e}}$ séance 10/02, article 14)

Des registres divers sont convoqués pour arguer de ce qui construit ou agit comme déterminisme pour un enfant $\left(\chi^{2}=45,82 ; 39,21\right)$ : l'amour, l'éducation, le patrimoine génétique, social et culturel transmis, son histoire, ses origines biologiques ( $\chi^{2}=80,58 ; 96,02 ; 38,71 ; 51,64 ; 73,29 ; 226,01$ ). En termes de température informationnelle, cela se traduit par une température élevée de la classe (indice de rareté le plus élevé des classes ; $\mathrm{IR}_{\mathrm{cl} 1}=029 \in[.017, .029]$; indice de diversité relativement élevé ; $\left.\mathrm{TTR}_{\mathrm{cl} 1}=.148 \in[.128, .169]\right)$. À noter, le relevé des mots-outils spécifiques de la classe indique une surreprésentation des verbes " savoir » et "falloir " $\left(\chi^{2}=18,87 ; 16,24\right)$. Ainsi, ce qui s'y joue est de l'ordre du normatif, et ce qu'il faut faire, selon les parlementaires, c'est définir le modèle de parenté, le 
schéma familial ( $\chi^{2}=28,64 ; 27,58$ ), c'est-à-dire décider des définitions de la famille et des fondements de la filiation ${ }^{8}$.

\section{Discussion et conclusion}

Cet article présente et discute les principaux résultats d'une étude psychosociale s'intéressant à la manière dont les parlementaires ont pensé la bioéthique et l'anonymat du don de gamètes, lors de la révision de la loi relative à la bioéthique en France en 2011. Ce faisant, cet article illustre la pertinence de l'analyse de cette arène de discussions lorsqu'il s'agit de saisir les modes de construction de la pensée sociale. Ces modes de construction ont été appréhendés en référence à la double fonction, instrumentale et communicative, des représentations sociales (Moscovici, 1976/2004). D'une part, la production et l'actualisation des représentations sociales peuvent s'observer dans les discussions parlementaires, dans une unité de lieux et de temps définie. Elle est assurée par la prescription institutionnelle qui est faite aux parlementaires de se familiariser avec un ensemble d'objets et de questions dilemmatiques, en les débattant, afin de se positionner à leur égard. Il est attendu que cette " mise en controverse " conduise à un environnement symbolique ambigu (Wagner et al., 2002), les représentations qui s'y échangent pouvant être décrites comme émancipées voire polémiques (Moscovici, 2013), et s'ancrant dans les groupes politiques. D'autre part, l'une des visées des discussions parlementaires peut être l'élaboration collective de représentations hégémoniques, structurantes pour le groupe-nation (Moscovici, 2013), dont la loi fera ensuite la promotion dans la sphère publique (Castro, 2012). Il est attendu que les parlementaires des différents groupes politiques s'appuient alors progressivement sur des éléments représentationnels davantage conventionnalisés, en vue de maintenir les conditions communes d'une communication possible, et afin de répondre à cet appel au consensus.

Une analyse lexicométrique (Ratinaud \& Marchand, 2015) d'un corpus regroupant les discussions générales et celles des articles de loi consacrés à la PMA, a permis de considérer, entre autres, cette première hypothèse diachronique selon laquelle l'organisation des discussions parlementaires serait marquée par un processus de conventionnalisation. Au sein de cette analyse, les mondes lexicaux ont aussi été comparés à l'aide d'indices relevant du lexiqueà même de renseigner leur température informationnelle. La pertinence de la prise en compte de ces indices s'appuie sur l'idée que la communication des représentations sociales ne laisse pas les mêmes traces indirectes sur le lexique, en fonction de leur utilisation à des fins instrumentales ou communicatives (Kalampalikis \& Moscovici, 2005). L'interprétation de la température informationnelle des mondes lexicaux, croisée avec l'analyse de leur contenu et les variables externes associées, peut ainsi participer judicieusement du repérage des éléments représentationnels émancipés voire polémiques (dont la communication est caractérisée par une haute température informationnelle) et des éléments hégémoniques ou conventionnalisés (dont la communication est caractérisée par une basse température informationnelle).

Les résultats indiquent que la classification se structure en deux pôles. Cela pourrait laisser envisager que deux manières de penser la bioéthique se sont dégagées. Or, l'examen des variables externes associées aux mondes lexicaux de chaque pôle montre que l'organisation en est plutôt gigogne, le premier pôle rassemblant des lieux lexicaux caractéristiques des discussions générales, le second recouvrant des articles de loi ayant trait à la PMA avec don de gamètes. Il y a un rapport d'antériorité du premier vis-à-vis du second, dans chaque Chambre parlementaire, correspondant à une structuration essentiellement institutionnelle des discussions. Notons également que la structuration de la classification est essentiellement non-partisane. Une coloration en termes d'appartenances aux groupes/partis politiques ne se retrouve que ponctuellement. Enfin, la structuration de la classification ne respecte pas non plus une chronologie des séances parlementaires qui aurait pu être la marque d'une dynamique représentationnelle « linéaire " vers des éléments représentationnels conventionnalisés, comme nous le supposions (elle aurait alors fait fi du passage des débats de l'Assemblée nationale au Sénat).

\footnotetext{
8 Il convient de rappeler que la question n'est pas ici légale, à savoir que le périmètre de la loi relative à la bioéthique n'inclut pas la question de la filiation juridique, définie par le Code civil. C'est bien d'une définition anthropologique de la parenté dont il s'agit.
} 
Cependant, l'hypothèse de discussions qui aboutissent à des représentations conventionnalisées ou hégémoniques est surtout battue en brèche du fait que les parlementaires aient initié leurs discussions par la recherche d'un cadre commun de communication, en dégageant des valeurs fondatrices consensuelles pour penser la bioéthique. L'interprétation du contenu et l'interprétation en termes de température informationnelle du premier monde lexical rendent compte de cette volonté des parlementaires d'inscrire l'action du législateur dans un cadre potentiellement fédérateur car supraordonné : la catégorie de pensée anthropologiquement fondamentale de l'humain a été mobilisée pour ce faire. Dans les discussions générales, la relation de l'humain et de la science a ainsi été traitée dans ses appréhensions les plus globalisantes. Deux acceptions de la science apparaissent, en ce qu'elle peut être conçue comme source d'humanisation, par le progrès scientifique, et de déshumanisation, par les technosciences. Dans la première acception, la science est spécifique à l'humain et ses progrès sont entendus comme des gains sur l'état de nature. Dans la seconde, la science a un potentiel réifiant, assimilant l'humain à du matériel biotechnique. Auquel cas, la liberté et l'autonomie individuelles et scientifiques sont à encadrer légalement pour garantir le respect de la dignité humaine. Alors que les principes de liberté et de dignité peuvent faire consensus considérés in abstracto, une cristallisation de cette tension se retrouve dans les distinctions faites entre les finalités scientifiques (ne pas entraver la recherche fondamentale) et les finalités thérapeutiques (bénéficier à la santé et à la vie) de la recherche sur l'embryon et les cellules embryonnaires (Alessio, Apostolidis, Santos, \& Dany, 2012). Cela pourrait expliquer le rapprochement des deux premiers lieux lexicaux de la classification (classes 1 et 6 ), alors que la recherche sur l'embryon est le seul point de décision spécifique à être inclus au sein du pôle relevant des discussions générales. Il n'en reste pas moins que le précepte de non-patrimonialité du corps humain, ainsi que le principe de " dignité humaine " au service duquel il est mobilisé (Ogien, 2012), se sont présentés comme hégémoniques dans les hémicycles français.

L'analyse des autres mondes lexicaux du premier pôle suggère que cet accord conventionnalisé avec ces valeurs génériques a servi de ressource face à une conflictualisation du débat, intervenue lors de la discussion générale à l'Assemblée nationale. Les résultats montrent que cette conflictualisation s'est fondée explicitement sur le refus du fait que les discussions bioéthiques représenteraient une exception dans l'exercice d'élaboration des lois en échappant aux dissensions politiques ou partisanes. La question de l'ancrage politique des représentations et des prises de position est lui-même devenu l'objet de la controverse. Des pratiques extérieures au périmètre de la loi relative à la bioéthique ont de plus été mentionnées comme des exemples historiques, pour asseoir l'argument selon lequel le consensus n'était pas nécessaire dans les chambres parlementaires pour conduire à un changement de la loi, lequel a été conventionnalisé ensuite dans la sphère publique. Nous interprétons le choix de certains de ces exemples (loi sur la fin de vie, avortement. ..) comme une référence à des débats emblématiques d'une tension entre les principes de liberté et d'autonomie individuelles, d'une part, et la recherche d'une adéquation entre la normativité juridique et les autres ordres normatifs (moral ou religieux), d'autre part. Cette recherche convoque le Droit sous une fonction anthropologique qui serait " celle d'instituer l'humain et d'être, alors, à la fois la limite et le fondement de sa liberté " (Hennette-Vauchez, 2009, p. 9). Face à cette tentative de dissension, une pression à l'uniformité s'est explicitée dans les discours parlementaires faisant appel à nouveau à des appartenances et des buts communs, transcendant les clivages partisans. Cet ancrage liminaire des discussions dans une catégorie de pensée relevant de l'universel a ainsi été utilisé comme stratégie de maintien du consensus dans la communication.

Le deuxième pôle de la classification relève des discussions des articles de lois relatifs à la PMA. Nous pourrions interpréter cette partition et le fait que l'appropriation symbolique de la bioéthique par les parlementaires soit marquée par des représentations hégémoniques dans sa première phase, comme le signe que l'on retrouve, au sein même du processus d'élaboration de la loi, des représentations qui deviennent davantage émancipées ou polémiques lorsqu'il s'agit de traduire les valeurs génériques en pratiques concrètes. Cette tension entre des représentations abstraites consensuelles, et des représentations concrètes polémiques, a été mise en évidence dans la phase de transition de l'innovation légale, lors de laquelle les groupes sociaux concernés tentent d'accommoder les savoirs globaux et génériques du nouveau cadre légal, dans leurs savoirs locaux, au sein de pratiques et de contextes spécifiques (Castro, 2012 ; Mouro \& Castro, 2012). Pour les discussions relatives à la 
bioéthique, cela aurait supposé que les parlementaires se soient accordés sur des principes communs lors des discussions générales, et que les déclinaisons pratiques de ces principes aient ensuite fait l'objet, dans les discussions des articles de loi, de polémiques et de divisions politiques. L'analyse des résultats ne soutient pas uniment cette idée. D'une part, la classification n'est pas structurée selon un ancrage des représentations dans les groupes politiques. Si des divergences de représentations et prises de position ont eu lieu entre les groupes politiques, elles ont été exprimées avec un lexique majoritairement commun. D'autre part, l'interprétation en termes de température informationnelle des mondes lexicaux du second pôle n'est probante pour y déceler des représentations que l'on pourrait décrire comme davantage polémiques, que croisée avec l'analyse de leur contenu.

L'analyse des formes et des segments de texte caractéristiques du monde lexical consacré à l'éligibilité au don de gamètes (classe 2) dénote ainsi une tension entre deux fragments de systèmes représentationnels (" éthique de l'autonomie " versus " éthique de la vulnérabilité ») qui apparaissait déjà à l'œuvre lors de la tentative de conflictualité politique des discussions, à l'Assemblée nationale. Ces systèmes représentationnels sont-ils également mobilisés par les parlementaires pour penser et se positionner quant à l'anonymat ou l'identification possible du donneur de gamètes ? C'est le cas lorsque " l'éthique de la vulnérabilité », désignée également comme « courant biologique " (Alessio et al., 2012: Fagot-Largeault, 2011), renvoie à une représentation philosophique et religieuse de l'humain où la nature se constitue en ordre moral à respecter. Dès lors, une prise de position en faveur de l'identification possible du donneur de gamètes, figurée comme un accès " aux origines ", et ancrée dans des théories biogénétiques de construction de la personne humaine, implique l'actualisation d'un tel système représentationnel. A contrario, des acceptions relevant d'une " éthique de l'autonomie " ne sont pas saillantes dans les mondes lexicaux consacrés, ni dans celui dévolu à la présentation de la question de l'anonymat du don ou de son identification possible, ni dans celui, accolé, traitant de la définition de la famille et des fondements de la filiation (classes 5 et 4 , respectivement). Cette proximité entre ces deux mondes lexicaux met en évidence que l'anonymat ou l'identification possible des donneurs de gamètes sont appropriés symboliquement en relation avec les cadres de pensée de la parenté et de la famille. Cette relation est d'autant plus signifiante qu'elle est mise en œuvre par des spécialistes de l'instauration des lois et que, précisément, la question de la filiation juridique ne se pose pas dans le cadre de la loi relative à la bioéthique. Ainsi, les discussions parlementaires sur le registre anonyme ou non des dons de gamètes apparaît s'être construit au regard des définitions génériques de la famille et de la filiation. À cet égard, Théry et Leroyer (2014) notent qu'elles considèrent que le régime d'anonymat du donneur est une question qui n'a pas été traitée durant le débat (public et parlementaire) à cause de la « confusion entre origines personnelles et filiation ». Deux traits fondamentaux du fonctionnement cognitif de la pensée sociale nous apparaissent à l'œuvre (Moscovici, 1976/2004). Un dualisme causal se retrouve dans l'ordonnancement qui est fait " par relation de voisinage » entre la possibilité d'une connaissance de l'identité du donneur et la création d'une relation filiale avec celuici, la détermination du sens de la relation causale étant énoncée dépendamment des intentions du groupe et des valeurs qu'il souhaite défendre (Moscovici, 1976/2004, p. 260). Une logique analogique (Moscovici, 1976/2004) a été, de plus, puissamment agissante, faisant de l'anonymat du donneur la partie protectrice d'un paradigme de la parenté, fondé sur l'exclusivité de celle-ci (les dimensions sociales et biogénétiques de la parenté y sont mutuellement exclusives). Trancher la question de l'identification possible du donneur de gamètes a ainsi été pensé par les parlementaires dans un rapport d'équivalence avec le fait de définir les contours normatifs, prévus pour être structurants pour tous, de ce que sont la famille, la parenté et la filiation.

L'analyse des débats parlementaires sur les questions bioéthiques illustre la pertinence de se pencher sur les matériaux qui les composent et les enjeux qui les gouvernent. Ces matériaux puisent majoritairement leurs fondements dans le sens commun : "C'est dans ce lieu épistémologique que sont venues s'inscrire les représentations sociales. Elles forment un ensemble riche de concepts et d'images liés entre eux, contrairement aux opinions, et s'entretissent au niveau du langage, des croyances mobiles quelle qu'en soit l'origine, dans la vie de tous les jours, l'épaisseur des relations familières, contrairement à l'idéologie " (Moscovici, 2019, p. 26). En matière bioéthique, l'évolution constante, technologique et sociale, de ces matériaux invite à examiner les relations constituées et constituantes entre les discussions parlementaires et d'autres formes de débats publics, ansi que les 
manifestations de ces relations à un niveau davantage micro-génétique en termes d'arguments au sein des discussions. En somme, il s'agirait d'intégrer en tant que telles les discussions parlementaires dans l'étude des innovations légales, et des résistances et changements associés (Castro, 2012). Nul doute que le changement légal annoncé par le vote des assemblées en faveur d'une levée possible de l'anonymat du donneur de gamètes lors de la dernière révision de la loi relative à la bioéthique en France en soit une occasion d'intérêt.

\section{Déclaration de liens d'intérêts}

Les auteurs déclarent ne pas avoir de liens d'intérêts.

\section{Financements}

Agence de la Biomédecine (PEGH 2010); région Auvergne Rhône-Alpes (CIBLE 2010).

\section{Annexe A. Matériel complémentaire}

Le matériel complémentaire (Appendice, Tableau 2) accompagnant la version en ligne de cet article est disponible sur http://www.sciencedirect.com et https://doi.org/10.1016/j.prps.2020.03.003.

\section{Références}

Alessio, R., Apostolidis, T., Santos, F., \& Dany, L. (2012). Représentations sociales et embryon humain : une étude comparative Brésil/France. Les Cahiers internationaux de psychologie sociale, 4, 371-395.

Bateman, S. (1994). Les passeurs de gamètes. Nancy: PUN.

Bateman, S. (1998). La bioéthique comme objet sociologique : figures de la connaissance. Cahiers internationaux de sociologie, $104,5-32$.

Castro, P. (2012). Legal innovation for social change: Exploring change and resistance to different types of sustainability laws. Political Psychology, 33, 105-121.

CCNE. (2018). Synthèse cartographique de la consultation publique. (Repéré à https://www.ccne-ethique.fr/)

Clémence, A., Doise, A., \& Lorenzi-Cioldi, F. (1994). Prises de position et principes organisateurs des représentations sociales. In C. Guimelli (Ed.), Structures et transformations des représentations sociales (pp. 119-152). Lausanne: Delachaux \& Niestlé.

Fagot-Largeault, A. (2011). Un regard de philosophe sur le statut de l'embryon et l'interruption volontaire de grossesse. Revue française des Affaires sociales, 1, 61-67.

Fox, R. C., \& Swazey, J. P. (2008). Observing bioethics. Oxford: Oxford University Press.

Gillespie, A. (2008). Social representations, alternative representations and semantic barriers. Journal for the Theory of Social Behaviour, 38(4), 375-391.

Hennette-Vauchez, S. (2009). Le droit de la bioéthique. Paris: La Découverte.

Jodelet, D. (2015). Représentations sociales et mondes de vie. Paris: Éditions des Archives contemporaines.

Jodelet, D. (2013). La rencontre des savoirs. Papers on Social Representations, 22 (9.1-9.20).

Kalampalikis, N. (2003). L'approche de la méthode Alceste dans l'analyse des représentations sociales. In J.-C. Abric (Ed.), Méthodes d'études des représentations sociales (pp. 147-163). Paris: Érès.

Kalampalikis, N., \& Moscovici, S. (2005). Une approche pragmatique de l'analyse Alceste. Les Cahiers internationaux de psychologie sociale, 66(2), 15-24.

Kronberger, N., \& Wagner, W. (2003). Keywords in context: Statistical analysis of text features. In M. Bauer, \& G. Gaskell (Eds.), Qualitative researching with text, image and sound (pp. 299-317). London: Sage.

Moscovici, S. (1967). Communication processes and the properties of language. In L. Berkowitz (Ed.), Advances in experimental social psychology (Vol. 3) (pp. 225-270). New York: Academic Press.

Moscovici, S. (1994). Social representations and pragmatic communication. Social Science Information, 33, 163-177.

Moscovici, S. (2004). La psychanalyse, son image et son public. Paris: PUF.

Moscovici, S. (1984). Psychologie sociale. Paris: PUF.

Moscovici, S. (2013). Le scandale de la pensée sociale. Paris: Éditions de l’Ehess.

Moscovici, S. (2019). Sens commun : représentations sociales ou idéologie ? In N. Kalampalikis (Ed.), Serge Moscovici : Psychologie des représentations sociales (pp. 17-29). Paris: Éditions des Archives contemporaines.

Mouro, C., \& Castro, P. (2012). Cognitive polyphasia in the reception of legal innovations for biodiversity conservation. Papers on Social Representations, 21(1) (1.1-1.21).

Ogien, R. (2012). La " marchandisation du corps humain ": les incohérences et les usages réactionnaires d'une dénonciation. Raison publique. http://www.raison-publique.fr/article534.html

Ratinaud, P. (2014). IRaMuTeQ : Interface de R pour les Analyses Multidimensionnelles de Textes et de Questionnaires. (Repéré à http://www.iramuteq.org/)

Ratinaud, P., \& Marchand, P. (2015). Des mondes lexicaux aux représentations sociales. Une première approche des thématiques dans les débats à l’Assemblée nationale (1998-2014). Mots, 2, 57-77.

Pour citer cet article : Doumergue, M., \& Kalampalikis, N. La fabrique parlementaire du discours bioéthique : analyse d'une arène de discussions. Pratiques psychologiques (2020), https://doi.org/10.1016/j.prps.2020.03.003 
Reinert, M. (1999). Quelques interrogations à propos de l'» objet » d'une analyse de discours de type statistique et de la réponse "Alceste ». Langage E' société, 90, 57-70.

Sebag-Depadt, V., \& Delaisi de Parseval, G. (2010). Accès à la parenté. Assistance médicale à la procréation et adoption pour une révision progressiste de la loi bioéthique. Paris: Fondation Terra Nova.

Théry, I. (2010). Des humains comme les autres. Bioéthique, anonymat et genre du don. Paris: Éditions de l'Ehess.

Théry, I., \& Leroyer, A. M. (2014). Filiation, origines, parentalité : le Droit face aux nouvelles valeurs de responsabilié générationnelle. Paris: Éditions Odile Jacob.

Van Dijk, T. A. (2003). Knowledge in parliamentary debates. Journal of language and politics, 2(1), 93-129.

Wagner, W., Kronberger, N., \& Seifert, F. (2002). Collective symbolic coping with new technology: Knowledge, images and public discourse. British Journal of Social Psychology, 41(3), 323-343. 